\title{
Cáncer de Senos Paranasales y sus Manifestaciones Orales: Reporte de Dos Casos Clínicos
}

\author{
Paranasal Sinus Cancer and Oral Manifestations: Report of Two Cases
}

Scarlette Hernández V.; Manuel Donoso Z.",*; Carlos Hernández A."* \& Luis Vásquez S."**

HERNÁNDEZ, V. S.; DONOSO, Z. M.; HERNÁNDEZ, A. C. \& VÁSQUEZ, S. L. Cáncer de senos paranasales y sus manifestaciones orales: reporte de dos casos clínicos. Int. J. Odontostomat., 7(1):53-57, 2013.

RESUMEN: Los carcinomas de senos paranasales son entidades poco frecuentes y cuando se diagnostican, generalmente ya se encuentran en estados avanzados de la enfermedad, dado que el proceso neoplásico en las etapas iniciales crece invadiendo las cavidades paranasales, para luego en etapas avanzadas producir la deformación de las corticales. Dentro de las manifestaciones clínicas se pueden presentar epistaxis, obstrucción nasal, dolor en zona maxilar, dolor o movilidad dentaria. Se presentan dos casos clínicos que dentro de las primeras manifestaciones cursaron con dolor de la zona maxilar y movilidad anormal de un diente. Al momento de realizar el diagnóstico definitivo los procesos tumorales se encontraban muy avanzados, comprometiendo diversas estructuras como seno maxilar, etmoidal, esfenoidal, y piso de órbita, lo que obliga a realizar grandes resecciones de la zona maxilofacial. El rol del odontólogo en el diagnóstico de la enfermedad podría ser de gran importancia, al sospechar que un aumento de volumen del reborde, la movilidad dentaria anormal o un dolor de la zona maxilar no siempre corresponden a causas odontogénicas.

PALABRAS CLAVE: cáncer de senos paranasales, carcinoma nasosinusal, neoplasias malignas.

\section{INTRODUCCIÓN}

Los carcinomas de senos maxilares son entidades de presentación poco frecuente, encontrándose alrededor de $0,2-0,8 \%$ de las neoplasias, un 3\% de los carcinomas de cabeza y cuello, y un $80 \%$ de los casos de tumores de senos paranasales (Quitral et al., 2003; Pires de Souza et al., 2006).

La mayoría de los pacientes presentan un estado avanzado de la enfermedad al momento de presentar el primer signo clínico, y cuando el tumor es de pequeño tamaño, puede ser mal diagnosticado como sinusitis crónica, pólipo nasal, obstrucción del ducto lagrimal. La tomografía computarizada (TC) y la resonancia magnética son técnicas útiles para evaluar la extensión del tumor en áreas adyacentes (Pires de Souza et al.). Aproximadamente el $85 \%$ de las neoplasias antromaxilares corresponden a carcinomas epidermoides, y un $5-15 \%$ a carcinoma adenoquístico (Pires de Souza et al.).

Entre el $40-60 \%$ de los casos presentan asimetrías faciales, aumentos de volumen en la cavidad oral, y extensión del tumor a la cavidad nasal. Estas lesiones pueden invadir la órbita, seno etmoidal, alvéolos dentales y paladar, fosa pterigopalatina y músculos pterigoideos. A través de la fosa pterigoides, puede extenderse hacia la fisura orbitaria y senos cavernosos (Pires de Souza et al.).

El objetivo de este trabajo es presentar dos casos de cáncer de senos paranasales, de diagnóstico tardío, los cuales presentaron sintomatología a nivel de la cavidad oral dentro de las primeras manifestaciones.

\section{REPORTE DE CASOS}

Caso Clínico 1. Mujer de 40 años de edad, proveniente de un sector rural. Acude a consultorio local por primera vez al presentar episodio de epistaxis, lugar donde se le realiza un taponamiento nasal anterior.

\footnotetext{
* Profesor Auxiliar, Instituto Odontoestomatología, Facultad de Medicina, Universidad Austral de Chile, Valdivia, Chile.

" Patólogo Oral, Hospital Base Valdivia, Valdivia, Chile. Profesor Auxiliar, Instituto de Odontoestomatología, Facultad de Medicina, Universidad Austral de Chile, Valdivia, Chile.

*** Otorrinolaringólogo, Hospital Base Valdivia, Valdivia, Chile.
} 
Desde esa fecha, continua con epistaxis intermitentes por fosa nasal derecha, que cedía habitualmente con maniobras caseras, pero que de igual modo la obliga a acudir en 4 oportunidades a su consultorio.

Tres meses después de la primera consulta, refiere dolor de zona molar superior derecha, oportunidad en que se realiza exodoncia del diente sospechoso de ser causal del dolor, además de indicar antibiótico inyectable. Posteriormente a este episodio, es derivada al centro de referencia regional, dado que la molestia dolorosa no cedía.

Ocho meses después de la primera consulta, acude al servicio de Otorrinolaringología, por continuar con episodios de hemorragias nasales a repetición. Se solicita TC de senos paranasales. Sin embargo, la paciente finalmente no se realiza dicho examen.

Catorce meses después del inicio de la sintomatología, es derivada al servicio dental del centro de referencia regional, desde consultorio rural, para

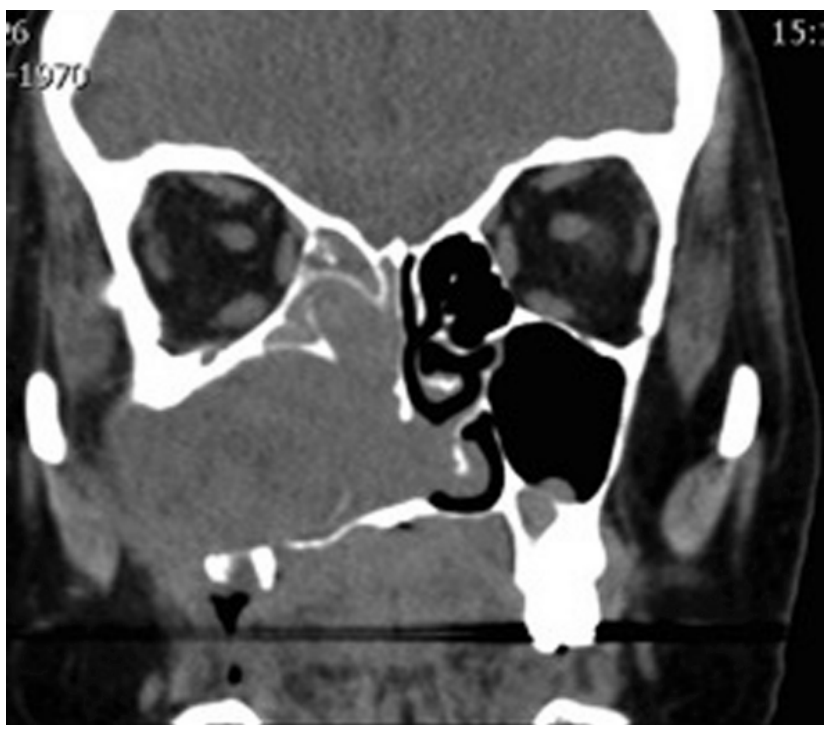

Fig. 1. Corte coronal con contraste TC.

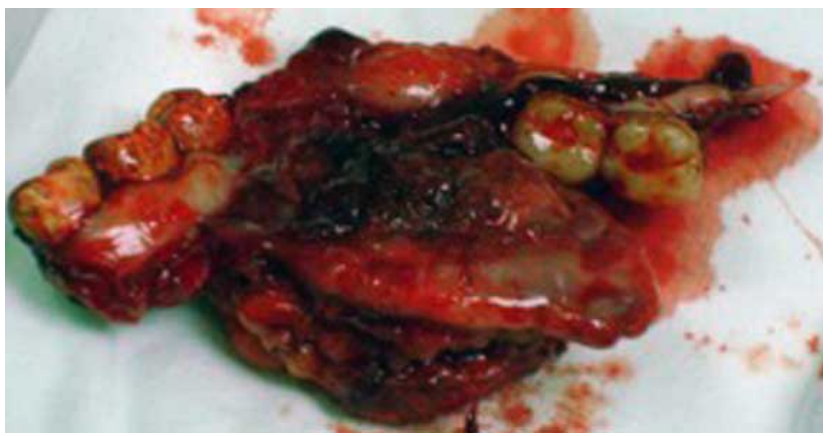

Fig. 2. Pieza operatoria. descartar el origen dentario en un posible cuadro de sinusitis maxilar. En el examen clínico, no se detecta pieza dentaria en mal estado, y es derivado nuevamente al Servicio de Otorrino, dado los antecedentes previos. La paciente nuevamente relata episodios de epistaxis a repetición por fosa nasal derecha con obstrucción respiratoria de dicho lado. En el examen clínico se constata presencia de una masa en la fosa nasal derecha y aumento de volumen en mejilla del mismo lado, razón por la cual, se toma biopsia de la tumoración nasal con anestesia local. Además, se indica TC de la zona.

Los resultados de TC de senos paranasales se observa la presencia de un proceso expansivo del seno maxilar y fosa nasal derecha, que compromete además, zona etmoidal anterior, posterior y seno esfenoidal derecho (Figs. 1 y 2). El resultado del examen histopatológico señala el diagnóstico de carcinoma escamoso de células grandes queratinizante moderadamente diferenciado. Se realiza estudio de diseminación regional y a distancia, lo cual fue descartado mediante TC de cuello y radiografía de torax. Con estos resultados es presentado al comité oncológico. Se programa cirugía, en la cual se realiza maxilectomía total derecha con etmoidectomía anterior y posterior. Resecando además, cavidad nasal derecha, concha nasal inferior y media, y seno esfenoidal derecho. Posteriormente, se realiza radioterapia complementaria.

Caso Clínico 2. Paciente sexo masculino, 73 años de edad, derivado de sector rural, consulta por aumento de volumen palatino y dolor en la zona maxilar derecha. Relata que comenzó con dolor 5 meses previo a la consulta en zona maxilar. Un mes después, comienza con aumento de volumen palatino, sin otro síntoma asociado.

Al momento del examen clínico presenta tumor doloroso del hemimaxilar derecho que expande corticales vestibular y palatina desde la tuberosidad hasta la zona canina derecha (Fig. 3). La superficie se presenta parcialmente ulcerada y fluctuante. Sin adenopatías asociadas.

El estudio imagenológico contempla radiografía panorámica, de Waters y TC. Además, de toma de biopsia incisional. El diagnóstico preliminar de la biopsia indica Carcinoma indiferenciado (Fig. 4).

Se somete la muestra a batería de inmunohistoquimica, cuyos resultados fueron Citoqueratina (+), HMB $45(-)$, S100 (-), Cromogranina A (-), que arrojaron como diagnóstico definitivo Carcinoma pobremente diferenciado, con acentuada necrosis. 


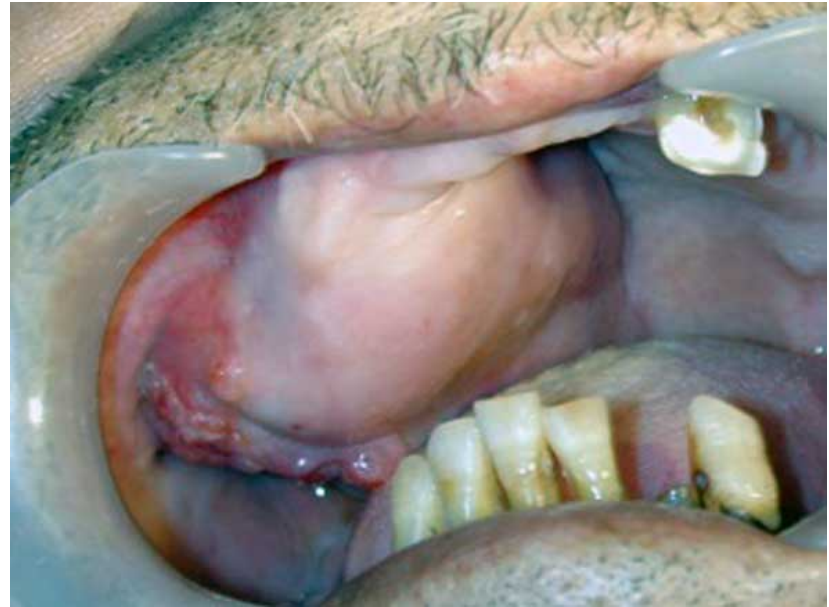

Fig. 3. Presentación clínica

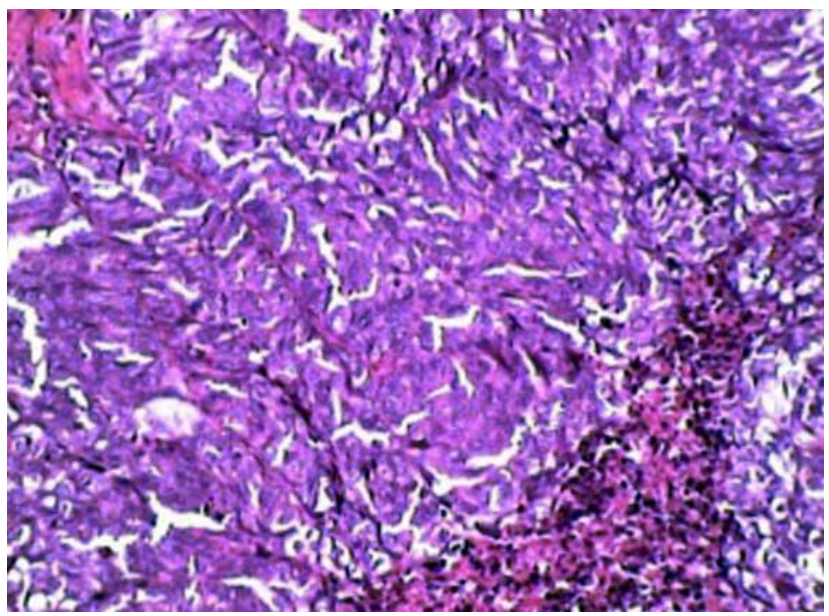

Fig. 4. Carcinoma indiferenciado. Biopsia preliminar. HE

Se indica tratamiento quirúrgico acompañado de radioterapia postoperatoria, sin embargo, el paciente y sus familiares, no aceptan la cirugía, y solicitan tratamiento paliativo.

\section{DISCUSIÓN}

Las neoplasias malignas sinusales son relativamente raras, y representan menos del $1 \%$ de los tumores malignos y un $3 \%$ de los provenientes del tracto respiratorio superior (Khademi et al., 2009; Rangel-Hernández et al., 2005).

La edad de presentación más frecuente es entre la $6^{a}$ y $7^{a}$ década de vida. Siendo extremadamente infrecuente su presentación antes de la segunda década de vida. (Khademi et al., 2009). En la mayoría de los estudios se señala la mayor frecuencia en hom- bres que en mujeres, sin embargo, en otros la proporción es prácticamente similar (Khademi et al.).

Dentro de la sintomatología que se presenta en estas tumoraciones, las más frecuentes son obstrucción nasal, dolor en zona premaxilar, epistaxis, dolor de cabeza, aumento de volumen facial, dolor dental entre otros (Panchal et al., 2005; Pires de Souza et al.; Quitral et al.).

Desde el punto de vista patológico se dividen en tumores de origen epitelial y no epitelial (RangelHernández \& Ordóñez-García, 2005). Los tumores de origen epitelial constituyen la mayoría de estas lesiones. Carcinomas de células escamosas, tumores de glándulas salivales menores y carcinomas indiferenciados son las neoplasias malignas más frecuentes en esta región (Khademi et al.). Los carcinomas constituyen casi dos tercios del total de estas neoplasias. De acuerdo a la literatura internacional, son más frecuentes los tumores benignos que los malignos, sin embargo, en una publicación mexicana, en dicha población se encontró una proporción invertida, siendo más frecuentes los malignos. (RangelHernández \& Ordóñez-García). En Chile, Quitral et al., en una revisión retrospectiva de 11 años, determino que el $32 \%$ de los casos de tumores malignos de senos paranasales corresponden a carcinoma de células escamosas.

El American Joint Committee on Cancer ha designado estadios para este tipo de cáncer, considerando el seno maxilar y etmoidal (Tabla I).

Dentro de las localizaciones más frecuentes se encuentran el seno maxilar, cavidad nasal, seguido de los senos etmoidal, esfenoidal y frontal (Khademi et al.).

El tratamiento de estas patologías difiere según el tipo de neoplasia, pero se basa en el tratamiento quirúrgico, quimioterapia y radioterapia (Khademi et al.; Gras Cabrerizo et al., 2007).

La afectación de linfonodos regionales es baja en estas neoplasias malignas, sin embargo, se considera realizar vaciamiento linfático regional en casos con afección alveolar, infiltración de tejidos blandos de mejilla, estados más avanzados o en casos cuya histología es desfavorable (Khademi et al.; Gras Cabrerizo et al.). La presencia de metástasis a distancia al momento del diagnóstico es baja, casi un $2 \%$ de los casos (Khademi et al.). La barrera más efectiva para la 
propagación del tumor sería la integridad del periostio, la cual es particularmente más resistente en dos áreas críticas, la base del cráneo y la órbita, lo que explica su comportamiento en esas zonas (Pires de Souza et al.).

Se han descrito diferentes factores carcinogénicos ambientales, biológicos y físicos. El consumo de alcohol y tabaco no representa una relación epidemiológica tan estrecha como con otros tumores de cabeza y cuello.

La relación con la exposición a polvo de maderas, curtidos, formaldehidos, polvo textil, asbesto o sícile se ha evidenciado. Además, se ha estudiado su relación con agentes virales como el virus papiloma humano o el virus Epstein Barr (Gras Cabrerizo et al.; Mannetje et al., 1999).

\section{CONCLUSIONES}

La dificultad del diagnóstico precoz es que el crecimiento tumoral se produce extendiéndose a través de las cavidades paranasales y se exterioriza cuando estas ya han sido ocupadas. Una de las zonas donde más frecuentemente se manifiesta su exteriorización es la cavidad oral, produciendo expansión de corticales, reborde maxilar, paladar duro, a veces acompañado de odontalgia, pudiendo producir incluso movilidad dentaria anormal.

Por lo anteriormente descrito, el papel del odontólogo en el diagnóstico de la enfermedad podría ser de gran importancia, al sospechar que un aumento de volumen del reborde o una odontalgia no siempre corresponden a causas odontogénicas.

Tabla I. Categorización de los cáncer de senos paranasales según criterios American Joint Committee on Cancer/ International union against cancer (1997) (Waldron \& Witterick, 2003).

\begin{tabular}{|c|c|c|}
\hline & Seno Maxilar & Seno Etmoidal \\
\hline T1 & Tumor se limita a la mucosa antral sin & Tumor confinado al etmoides con o sin erosión \\
\hline T2 & $\begin{array}{l}\text { erosión ni destrucción ósea. } \\
\text { El tumor causa erosión o destrucción ósea, } \\
\text { excepto por la pared posterior del seno }\end{array}$ & $\begin{array}{l}\text { ósea. } \\
\text { Tumor se extiende a la cavidad nasal. }\end{array}$ \\
\hline & $\begin{array}{l}\text { maxilar, incluye extensión al paladar duro y/o } \\
\text { meato nasal medio. }\end{array}$ & \\
\hline T3 & $\begin{array}{l}\text { El tumor invade algo de lo siguiente: pared } \\
\text { ósea posterior del seno maxilar, tejido } \\
\text { subcutáneo, piel de la mejilla, piso o pared } \\
\text { medial de la órbita, fosa infratemporal, placa }\end{array}$ & $\begin{array}{l}\text { El tumor se extiende a la zona anterior de la } \\
\text { órbita, y/o seno maxilar. }\end{array}$ \\
\hline T4 & $\begin{array}{l}\text { Tumor invade el contenido orbitario más allá } \\
\text { del piso o de la pared medial, incluyendo } \\
\text { alguna de las siguientes estructuras: ápice } \\
\text { orbital, placa cribiforme, base del cráneo, } \\
\text { nasofaringe, esfenoides, seno frontal. }\end{array}$ & $\begin{array}{l}\text { Tumor presenta extensión intracraneal, } \\
\text { extensión orbitaria incluyendo el vértice, } \\
\text { esfenoides, y/o seno frontal, y/o piel de la nariz }\end{array}$ \\
\hline
\end{tabular}

HERNÁNDEZ, V. S.; DONOSO, Z. M.; HERNÁNDEZ, A. C. \& VÁSQUEZ, S. L. Paranasal sinus cancer and oral manifestations: Report of two cases. Int. J. Odontostomat., 7(1):53-57, 2013.

ABSTRACT: Paranasal sinus cancer are rare entities, but when diagnosed, often already in advanced stages of the disease, because the neoplastic process in the initial stages grows invading the paranasal sinuses and then in advanced stages, produces deformation of cortical bone. Among the clinical manifestations epistaxis, nasal obstruction, pain in maxillary area, pain or tooth mobility may occur. We present two clinical cases in which within the first manifestations are the maxillary pain and abnormal tooth mobility. When making the final diagnosis, tumor processes were well advanced, engaging various structures as maxillary sinus, ethmoid, sphenoid, and orbital floor, forcing large resections perform maxillofacial area. The role of the dentist in the diagnosis of the disease could be of great importance, suspecting that an increase maxillary volume, abnormal tooth mobility or pain of the maxillary not always correspond with odontogenic causes.

KEY WORDS: paranasal sinus cancer, sinonasal carcinoma, malignant neoplasms. 
HERNÁNDEZ, V. S.; DONOSO, Z. M.; HERNÁNDEZ, A. C. \& VÁSQUEZ, S. L. Cáncer de senos paranasales y sus manifestaciones orales: reporte de dos casos clínicos. Int. J. Odontostomat., 7(1):53-57, 2013.

\section{REFERENCIAS BIBLIOGRÁFICAS}

Gras Cabrerizo, J. R.; Sarandeses García, A.; Montserrat, I.; Gili, J. R. \& Orús Dotú, C. Revisión de los carcinomas de senos paranasales. Acta Otorrinolaringol. Esp., 58(6):266-75, 2007.

Khademi, B.; Moradi, A.; Hoseini, S. \& Mohammadianpanah, M. Malignant neoplasms of the sinonasal tract: report of 71 patients and literature review and analysis. Oral Maxillofac. Surg., 13(4):191-9, 2009.

Panchal, L.; Vaideeswar, P.; Kathpal, D.; Madiwale, C. V. \& Prabhat, D. P. Sino-nasal epithelial tumours: a pathological study from 69 cases. J. Postgrad. Med., 51(1):30-4, 2005.

Pires de Souza, R.; Barros Cordeiro, F.; Mota Gonzalez, F.; Yamashiro, I.; Oliveira Paes Junior, A. J.; Souza Tornin, O.; et al. Maxillary sinus carcinoma: An analysis of ten cases. Radiol. Bras., 39(6):397-400, 2006.

Quitral, C. R.; Rahal, E. M.; Morales, G. I.; Daszenies, S. C.; Vallejos, U. M. P. Tumores malignos de nariz y cavidades paranasales. Revisión de 11 años. Rev. Otorrinolaringol. Cir. Cabeza Cuello, 63:21-8, 2003.

Rangel-Hernández, M. \& Ordóñez-García, R. Prevalencia de tumoraciones de nariz y senos paranasales en el Centro Médico Nacional "20 de Noviembre" de enero de 1994 a diciembre de 2002. AN. ORL. MEX., 50(1):3642, 2005.

't Mannetje, A.; Kogevinas, M.; Luce, D.; Demers, P. A.; Bégin, D.; Bolm-Audorff, U.; et al. Sinonasal cancer, occupation, and tobacco smoking in European women and men. Am. J. Ind. Med., 36(1):101-7, 1999.

Waldron, J. \& Witterick, I. Paranasal sinus cancer: caveats and controversies. World J. Surg., 27(7):849-55, 2003.
Dirección para Correspondencia: Scarlette Hernández Vigueras Instituto de Odontoestomatología Rudloff 1640

Valdivia

CHILE

Email: scarlettehv@gmail.com

Recibido : 02-10-2012

Aceptado: 29-01-2013 